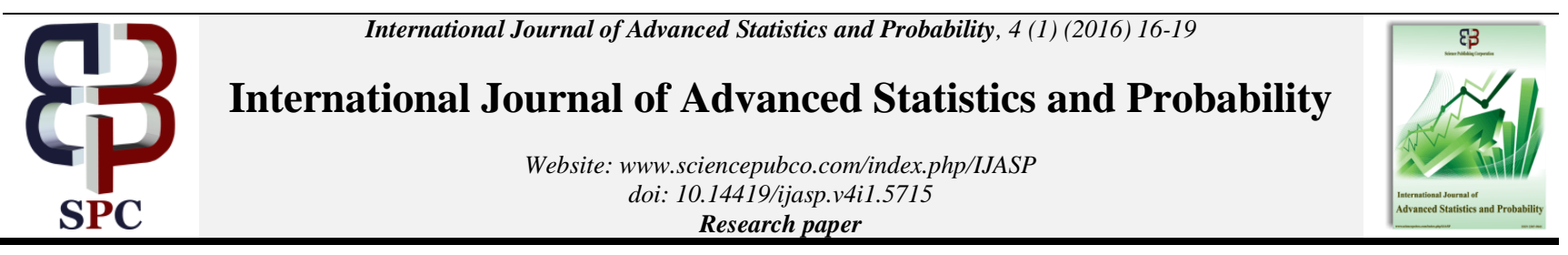

\title{
Two fixed point theorems in generalized metric spaces
}

\author{
Salwa Salman Abed *, Hadeel Hussein Luaibi \\ Department of Mathematics, College of Pure Science - Ibn Al-Haitham, Baghdad University \\ *Corresponding authorE-mail: salwaalbundi@yahoo.com
}

\begin{abstract}
In this paper, we prove there exists a coupled fixed point for a set- valued contraction mapping defined on $\mathrm{X} \times \mathrm{X}$, where $\mathrm{X}$ is incomplete ordered G-metric. Also, we prove the existence of a unique fixed point for single valued mapping with respect to implicit condition defined on a complete G- metric.
\end{abstract}

Keywords: G-Metric Spaces; Fixed Points; Coupled Fixed Points; Implicit Conditions.

\section{Introduction}

Nadler [1] initiated the study of fixed points for multi-valued contraction mappings and generalized the well-known Banach's fixed point principle. And then, many authors studied many fixed point results for multi-valued contraction mappings, see [2-3].

Mustafa and Sims [4] introduced the G-metric spaces as a generalization of the notion of metric spaces. Mustafa et al. [5- 6] obtained some fixed point theorems for mappings satisfying different contractive conditions. Abbas and Rhoades [7] initiated the study of common fixed point in G-metric spaces. While, Saadati et al. [8] studied some fixed point theorems in generalized partially ordered G-metric spaces.

Ran and Reurings [9] extended Banach's principle in partially ordered metric space to obtained some fixed point results for set valued mappings where the contraction condition is assumed only for the comparable elements of the partially ordered set.

In[10], Bhaskar and Lakshmikantham introduced the notions of mixed monotone property and coupled fixed point for the contractive mapping $\mathrm{F}: \mathrm{X} \times \mathrm{X} \rightarrow \mathrm{X}$, where $\mathrm{X}$ is a partially ordered metric space, and proved some coupled fixed point theorems for a mixed monotone operator. As an application of the coupled fixed point theorems, they determined the existence and uniqueness of the solution of a periodic boundary value problem. Recently, Lakshmikantham and Ćirić [11] have proved coupled coincidence and coupled common fixed point theorems for nonlinear contractive mappings in partially ordered complete metric spaces.

In this paper, firstly, we prove coupled fixed point theorem for setvalued mappings by weaken the hypotheses and replace the competence by some other conditions depending on ordering. Secondly, we prove the existence of unique fixed point for single valued mappings in incomplete G- metric space by using implicit condition. Throughout this paper $2^{\mathrm{X}}$ is the class of all non-empty subsets of $\mathrm{X}, \mathrm{CB}(\mathrm{X})$ is the class of all closed and bounded subsets of $\mathrm{X}$ and " $\rightarrow$ "denote to set-valued mappings.

\section{Preliminaries}

We begin with some basic definitions and facts.
Definition 2.1: [4] Let $\mathrm{X}$ be a non-empty set and G: $\mathrm{X} \times \mathrm{X} \times \mathrm{X} \rightarrow$ $[0,+\infty)$ be a function for all $x, y, z, a$ in $X$ satisfying the following conditions:

1) $G(x, y, z)=0 \Leftrightarrow x=y=z$

2) $0<G(x, x, y)$ with $x \neq y$

3) $G(x, x, y) \leq G(x, y, z)$ with $y \neq z$

4) $G(x, y, z)=G(p(x, z, y)), p(x, y, z)$ is a permutation of $x, y, z$

5) $G(x, y, z) \leq G(x, a, a)+G(a, y, z)$.

Then the ordered pair $(X, G)$ is called a generalized metric space or G- metric space.

Example 2.2: [12] Consider $X=R$, with usual distance $\mathrm{d}(\mathrm{x}, \mathrm{y})=|\mathrm{x}-\mathrm{y}|$, for all $\mathrm{x}, \mathrm{y}$ in $\mathrm{X}$. Define $\mathrm{G}: \mathrm{X}^{3} \rightarrow \mathrm{R}^{+}$by

$$
G(x, y, z)=d(x, y)+d(y, z)+d(z, x), \text { for all } x, y, z \in X .
$$

Then, $\mathrm{X}$ is a G-metric space.

Definition 2.3: [4] Let $(X, G)$ be a $G$ - metric space. The sequence $\left\{x_{n}\right\}$ is called

1) A G-Cauchy if, $\forall \varepsilon>0$, there is $\mathrm{k} \in \mathrm{N}$ such that for all positive integers $n, m, 1 \geq k, G\left(x_{n}, x_{m}, x_{l}\right)<\varepsilon$

2) A G-convergent to $x \in X$ if, $\forall \varepsilon>0$, there is $k \in N$ such that for all $n, m \geq k, G\left(x, x_{n}, x_{m}\right)<\varepsilon$.

Also, $(X, G)$ is said to be complete G-metric space if every GCauchy sequence in $\mathrm{X}$ is $\mathrm{G}$ - convergent in $\mathrm{G}$.

A modification for the Husdorff distance is:

Definition 2.4: [13] Let $X$ be a G-metric space. $H$ is called the Hausdorff G- distance on $\mathrm{CB}(\mathrm{X})$, if

$H_{G}(A, B, C)=\max \left\{\sup _{x \in A} G(x, B, C), \sup _{x \in B} G(x, C, A), \sup _{x \in C} G(x, A\right.$, B) \},

Where

$\mathrm{G}(\mathrm{x}, \mathrm{B}, \mathrm{C})=\mathrm{d}_{\mathrm{G}}(\mathrm{x}, \mathrm{B})+\mathrm{d}_{\mathrm{G}}(\mathrm{B}, \mathrm{C})+\mathrm{d}_{\mathrm{G}}(\mathrm{x}, \mathrm{C})$,

$d_{G}(x, B)=\inf \left\{d_{G}(x, y), y \in B\right\}$,

$d_{G}(A, B)=\inf \left\{d_{G}(a, b), a \in A, b \in B\right\}$. 
Lemma 2.5: [13] If $A, B \in C B(X)$ and $a \in A$, then for each $\varepsilon>0$, there exists $b \in B$ such that

$$
\mathrm{G}(\mathrm{a}, \mathrm{b}, \mathrm{b}) \leq \mathrm{H}_{\mathrm{G}}(\mathrm{A}, \mathrm{B}, \mathrm{B})+\varepsilon
$$

Lemma 2.6: Let $A \in C B(X)$ and $B \in K(X)$ then for any a $\in A$ there is $b \in B$ such that:

$$
G(a, b, b) \leq H_{G}(A, B, B) \text {. }
$$

Lemma 2.7: Let $X$ be a $G$-metric space, if $A, B$ and $C \in C B(X)$ with $H_{G}(A, B, C) \leq \epsilon$, then for each a $\in A$ there exist elements $b \in$ $B, c \in C$ such that $G(a, b, c) \leq \epsilon$.

Lemma 2.8: Let $X$ be a $G$-metric space, and $\left\{A_{n}\right\}$ be sequence in $C B(X)$ and $\lim _{n \rightarrow \infty} H_{G}\left(A_{n}, A, A\right)=0$ for $A \in C B(X)$. If $x_{n} \in A_{n}$ and $\lim _{n \rightarrow \infty} G\left(x_{n}, x, x\right)=0$, then $x \in A$.

Proof: It is enough to prove that $G\left(x_{n}, A_{n}, A_{n}\right) \rightarrow G(x, A, A)$.

Definition 2.9: [14] Let $(X, \preccurlyeq)$ be a partially ordered set. Then $x, y \in X$ are called comparable if $x \leqslant y$ or $y \leqslant x$ holds.

Definition 2.10: [13] The point $x$ in $X$ is called a fixed point of the multivalued mapping $T: X \sim 2^{x}$ if $x \in T x$ and $x$ is fixed point of a single mapping $T: X \rightarrow X$ if $x=T x$.

Definition 2.11: An element $(x, y) \in X \times X$ is called a coupled fixed point of mapping $T: X \times X \leadsto 2^{X}$ if $x \in T(x, y)$ and $y \in T(y, x)$.

The following lemmas are needed:

\section{Coupled fixed point}

Theorem 3.1: Let $X$ is ordered G-metric space and let $T$ : $X \times X$ $\neg \mathrm{K}(\mathrm{X})$ satisfying:

1) There exists $\mathrm{k} \in(0,1)$ with

$\mathrm{H}_{\mathrm{G}}(\mathrm{T}(\mathrm{x}, \mathrm{y}), \mathrm{T}(\mathrm{u}, \mathrm{v}), \mathrm{T}(\mathrm{w}, \mathrm{z})) \leq \frac{\mathrm{k}}{2} \mathrm{G}((\mathrm{x}, \mathrm{y}),(\mathrm{u}, \mathrm{v}),(\mathrm{w}, \mathrm{z}))$

For all $(w, z) \preccurlyeq(u, v) \preccurlyeq(x, y)$.

2) Let $\mathrm{u}_{1} \in \mathrm{T}\left(\mathrm{x}_{1}, \mathrm{y}_{1}\right), \mathrm{v}_{1} \in \mathrm{T}\left(\mathrm{y}_{1}, \mathrm{x}_{1}\right)$ If $\mathrm{x}_{2} \preccurlyeq \mathrm{x}_{3}, \mathrm{y}_{3} \preccurlyeq \mathrm{y}_{2}, \mathrm{x}_{\mathrm{i}}, \mathrm{y}_{\mathrm{i}} \in$ $X(i=2,3)$ then for all $u_{2} \in T\left(x_{2}, y_{2}\right)$ there exists $u_{3} \in T\left(x_{3}\right.$, $\left.\mathrm{y}_{3}\right)$ with $\mathrm{u}_{2} \preccurlyeq \mathrm{u}_{3}$, and for all $\mathrm{v}_{1} \in \mathrm{T}\left(\mathrm{y}_{1}, \mathrm{x}_{1}\right)$ there exists $\mathrm{v}_{2} \in$ $\mathrm{T}\left(\mathrm{y}_{2}, \mathrm{x}_{2}\right)$ with $\mathrm{v}_{3} \preccurlyeq \mathrm{v}_{2}$ provided $\mathrm{G}\left(\left(\mathrm{u}_{1}, \mathrm{v}_{1}\right),\left(\mathrm{u}_{2}, \mathrm{v}_{2}\right),\left(\mathrm{u}_{3}\right.\right.$, $\left.\left.v_{3}\right)\right)<1$

3) There exist $x_{0}, y_{0} \in X$, and some $x_{1} \in T\left(x_{0}, y_{0}\right), y_{1} \in$ $\mathrm{T}\left(\mathrm{y}_{0}, \mathrm{x}_{0}\right), \mathrm{x}_{2} \in \mathrm{T}\left(\mathrm{x}_{1}, \mathrm{y}_{1}\right), \mathrm{y}_{1} \in \mathrm{T}\left(\mathrm{y}_{0}, \mathrm{x}_{0}\right)$ with $\mathrm{x}_{0} \preccurlyeq \mathrm{x}_{1} \preccurlyeq \mathrm{x}_{2}$ , $\mathrm{y}_{2} \preccurlyeq \mathrm{y}_{1} \preccurlyeq \mathrm{y}_{0}$ such that $\mathrm{G}\left(\left(\mathrm{x}_{0}, \mathrm{y}_{0}\right),\left(\mathrm{x}_{1}, \mathrm{y}_{1}\right),\left(\mathrm{x}_{2}, \mathrm{y}_{2}\right)\right)<1$ $\mathrm{k}$, wherek $\in(0,1)$.

4) If a non-decreasing sequence $x_{n} \rightarrow x$ in $X$ then $x_{n} \preccurlyeq x$, for all $\mathrm{n}$ and if a non-increasing sequence $\mathrm{y}_{\mathrm{n}} \rightarrow \mathrm{y}$ in $\mathrm{X}$, then $\mathrm{y} \preccurlyeq$ $\mathrm{y}_{\mathrm{n}}$, for all $\mathrm{n}$

Then $\mathrm{T}$ has a coupled fixed point.

Proof: Let $x_{0}, y_{0} \in X$ then by hypotheses (3) there exists $x_{1} \in$ $\mathrm{T}\left(\mathrm{x}_{0}, \mathrm{y}_{0}\right), \mathrm{y}_{1} \in \mathrm{T}\left(\mathrm{y}_{0}, \mathrm{x}_{0}\right), \mathrm{x}_{2} \in \mathrm{T}\left(\mathrm{x}_{1}, \mathrm{y}_{1}\right), \mathrm{y}_{2} \in \mathrm{T}\left(\mathrm{y}_{1}, \mathrm{x}_{1}\right)$ with $\mathrm{x}_{0} \preccurlyeq$ $\mathrm{x}_{1} \preccurlyeq \mathrm{x}_{2}, \mathrm{y}_{2} \preccurlyeq \mathrm{y}_{1} \preccurlyeq \mathrm{y}_{0}$ such that

$$
\mathrm{G}\left(\left(\mathrm{x}_{0}, \mathrm{y}_{0}\right),\left(\mathrm{x}_{1}, \mathrm{y}_{1}\right),\left(\mathrm{x}_{2}, \mathrm{y}_{2}\right)\right)<1-\mathrm{k} .
$$

Since $\left(\mathrm{x}_{0}, \mathrm{y}_{0}\right) \preccurlyeq\left(\mathrm{x}_{1}, \mathrm{y}_{1}\right) \preccurlyeq\left(\mathrm{x}_{2}, \mathrm{y}_{2}\right)$.By using assumption (1) and (3.1), we have

$\mathrm{H}_{\mathrm{G}}\left(\mathrm{T}\left(\mathrm{x}_{0}, \mathrm{y}_{0}\right), \mathrm{T}\left(\mathrm{x}_{1}, \mathrm{y}_{1}\right), \mathrm{T}\left(\mathrm{x}_{2}, \mathrm{y}_{2}\right)\right) \leq \frac{\mathrm{k}}{2} \mathrm{G}\left(\left(\mathrm{x}_{0}, \mathrm{y}_{0}\right),\left(\mathrm{x}_{1}, \mathrm{y}_{1}\right),\left(\mathrm{x}_{2}, \mathrm{y}_{2}\right)\right)$

$$
<\frac{\mathrm{k}}{2}(1-\mathrm{k})
$$

And similarly

$$
\mathrm{H}_{\mathrm{G}}\left(\mathrm{T}\left(\mathrm{y}_{0}, \mathrm{x}_{0}\right), \mathrm{T}\left(\mathrm{y}_{1}, \mathrm{x}_{1}\right), \mathrm{T}\left(\mathrm{y}_{2}, \mathrm{x}_{2}\right)\right)<\frac{\mathrm{k}}{2}(1-\mathrm{k})
$$

Using assumption (2) and lemma (2.7), there exist $\mathrm{x}_{3} \in \mathrm{T}\left(\mathrm{x}_{2}, \mathrm{y}_{2}\right)$, $\mathrm{y}_{3} \in \mathrm{T}\left(\mathrm{y}_{2}, \mathrm{x}_{2}\right)$ with $\mathrm{x}_{2} \preccurlyeq \mathrm{x}_{3}$ and $\mathrm{y}_{3} \preccurlyeq \mathrm{y}_{2}$ such that

$$
\mathrm{G}\left(\mathrm{x}_{1}, \mathrm{x}_{2}, \mathrm{x}_{3}\right) \leq \frac{\mathrm{k}}{2}(1-\mathrm{k}) \quad \cdots
$$

and

$$
\mathrm{G}\left(\mathrm{y}_{1}, \mathrm{y}_{2}, \mathrm{y}_{3}\right) \leq \frac{\mathrm{k}}{2}(1-\mathrm{k}) \quad \ldots
$$

From (3.2) and (3.3)

$$
\mathrm{G}\left(\left(\mathrm{x}_{1}, \mathrm{y}_{1}\right),\left(\mathrm{x}_{2}, \mathrm{y}_{2}\right),\left(\mathrm{x}_{3}, \mathrm{y}_{3}\right)\right) \leq \mathrm{k}(1-\mathrm{k}) \quad \ldots
$$

Again by assumption (1) and (3.4), we have

$$
\mathrm{H}_{\mathrm{G}}\left(\mathrm{T}\left(\mathrm{x}_{1}, \mathrm{y}_{1}\right), \mathrm{T}\left(\mathrm{x}_{2}, \mathrm{y}_{2}\right), \mathrm{T}\left(\mathrm{x}_{3}, \mathrm{y}_{3}\right)\right) \leq \frac{\mathrm{k}^{2}}{2}(1-\mathrm{k})
$$

and

$$
\mathrm{H}_{\mathrm{G}}\left(\mathrm{T}\left(\mathrm{y}_{1}, \mathrm{x}_{1}\right), \mathrm{T}\left(\mathrm{y}_{2}, \mathrm{x}_{2}\right), \mathrm{T}\left(\mathrm{y}_{3}, \mathrm{x}_{3}\right)\right) \leq \frac{\mathrm{k}^{2}}{2}(1-\mathrm{k})
$$

Further from lemma (2.7) and assumption (2), there exist $\mathrm{x}_{4} \in$ $\mathrm{T}\left(\mathrm{x}_{3}, \mathrm{y}_{3}\right), \mathrm{y}_{4} \in \mathrm{T}\left(\mathrm{y}_{3}, \mathrm{x}_{3}\right)$ with $\mathrm{x}_{3} \preccurlyeq \mathrm{x}_{4}, \mathrm{y}_{4} \preccurlyeq \mathrm{y}_{3}$ such that

$$
G\left(x_{2}, x_{3}, x_{4}\right) \leq \frac{k^{2}}{2}(1-k) .
$$

and

$$
\mathrm{G}\left(\mathrm{y}_{2}, \mathrm{y}_{3}, \mathrm{y}_{4}\right) \leq \frac{\mathrm{k}^{2}}{2}(1-\mathrm{k})
$$

It follows that

$$
G\left(\left(x_{2}, y_{2}\right),\left(x_{3}, y_{3}\right),\left(x_{4}, y_{4}\right)\right) \leq k^{2}(1-k) .
$$

Continue in this way, we obtain $x_{n+2} \in T\left(x_{n+1}, y_{n+1}\right), y_{n+2} \in$ $\mathrm{T}\left(\mathrm{y}_{\mathrm{n}+1}, \mathrm{x}_{\mathrm{n}+1}\right)$ with $\mathrm{x}_{\mathrm{n}+1} \preccurlyeq \mathrm{x}_{\mathrm{n}+2}, \mathrm{y}_{\mathrm{n}+2} \preccurlyeq \mathrm{y}_{\mathrm{n}+1}$ such that

$$
\mathrm{G}\left(\mathrm{x}_{\mathrm{n}}, \mathrm{x}_{\mathrm{n}+1}, \mathrm{x}_{\mathrm{n}+2}\right) \leq \frac{\mathrm{k}^{\mathrm{n}}}{2}(1-\mathrm{k})
$$

and

$$
G\left(y_{n}, y_{n+1}, y_{n+2}\right) \leq \frac{k^{n}}{2}(1-k)
$$

thus

$$
G\left(\left(x_{n}, y_{n}\right),\left(x_{n+1}, y_{n+1}\right),\left(x_{n+2}, y_{n+2}\right)\right) \leq k^{n}(1-k) \ldots
$$

Next, we show that $\left\{x_{n}\right\}$ is a G-Cauchy sequence in $X$. Let $m>n$. Then

$\mathrm{G}\left(\mathrm{x}_{\mathrm{n}}, \mathrm{x}_{\mathrm{n}+1}, \mathrm{x}_{\mathrm{m}}\right) \leq \mathrm{G}\left(\mathrm{x}_{\mathrm{n}}, \mathrm{x}_{\mathrm{n}+1}, \mathrm{x}_{n+1}\right)+G\left(x_{n+1}, x_{n+1}, x_{m}\right) \leq G\left(x_{n}\right.$, $\left.x_{n+1}, x_{n+2}\right)+G\left(x_{n+1}, x_{n+2}, x_{m}\right)$

[by using definition (2.1-3)]

$$
\leq G\left(x_{n}, x_{n+1}, x_{n+2}\right)+G\left(x_{n+1}, x_{n+2}, x_{n+2}\right)+G\left(x_{n+2}, x_{n+2}, x_{m}\right)
$$

[by using definition (2.1-5)]

$$
\leq G\left(x_{n}, x_{n+1}, x_{n+2}\right)+G\left(x_{n+1}, x_{n+2}, x_{n+3}\right)+G\left(x_{n+2}, x_{n+3}, x_{m}\right)
$$

[by using definition (2.1-3)]

$\leq G\left(x_{n}, x_{n+1}, x_{n+2}\right)+G\left(x_{n+1}, x_{n+2}, x_{m}\right)+G\left(x_{n}, x_{n+1}, x_{n+1}\right)+\ldots+$

$$
G\left(x_{m-2}, x_{m-1}, x_{m}\right)
$$




$$
\begin{aligned}
& \leq\left[k^{n}+k^{n+1}+\ldots+k^{m-2}\right] \frac{(1-k)}{2} \\
& =k^{n}\left[1+\mathrm{k}+\ldots+k^{m-n-2}\right] \frac{(1-k)}{2} \\
& =k^{n}\left[\frac{\left(1-k^{m-n-1}\right)}{1-k}\right] \frac{(1-k)}{2} \\
& =\frac{k^{n}}{2}\left(1-k^{m-n-1}\right)<\frac{k^{n}}{2},
\end{aligned}
$$

Since $\mathrm{k} \in(0,1), 1-k^{m-n-1}<1$, we obtain $G\left(x_{n}, x_{n+1}, x_{m}\right) \rightarrow 0$ as $\mathrm{n} \rightarrow \infty$. This implies that $\left\{x_{n}\right\}$ is a G-Cauchy sequence and hence converges to some point $\mathrm{x}$ in the complete in $\mathrm{X}$.

Similarly, we can show that $\left\{y_{n}\right\}$ is also a G-Cauchy sequence in $\mathrm{X}$.By completeness of $\mathrm{X}$, there exist $\mathrm{x}, \mathrm{y} \in \mathrm{X}$ such that $x_{n} \rightarrow \mathrm{x}$ and $y_{n} \rightarrow \mathrm{y}$ as $\mathrm{n} \rightarrow \infty$.

Finally, we show that $\mathrm{x} \in \mathrm{T}(\mathrm{x}, \mathrm{y})$ and $\mathrm{y} \in \mathrm{T}(\mathrm{y}, \mathrm{x})$.

Since $\left\{x_{n}\right\}$ is a non-decreasing sequence and $\left\{y_{n}\right\}$ is a nonincreasing sequence in $\mathrm{X}$ such that $x_{n} \rightarrow \mathrm{x}$ and $y_{n} \rightarrow \mathrm{y}$, therefore we have $x_{n} \preccurlyeq \mathrm{x}, \mathrm{y} \preccurlyeq y_{n}$ for all $\mathrm{n}$.

From hypothesis (1) it follows that

$$
\mathrm{H}_{\mathrm{G}}\left(\mathrm{T}\left(x_{n}, y_{n}\right), \mathrm{T}(\mathrm{x}, \mathrm{y}), \mathrm{T}(\mathrm{x}, \mathrm{y})\right) \leq \mathrm{k} G\left(\left(x_{n}, y_{n}\right),(\mathrm{x}, \mathrm{y}),(\mathrm{x}, \mathrm{y})\right) \rightarrow 0
$$

Now, since $x_{n+1} \in \mathrm{T}\left(x_{n}, y_{n}\right)$ and $\lim _{\mathrm{n} \rightarrow \infty} G\left(x_{n+1}, \mathrm{x}, \mathrm{x}\right)=0$, it then by using lemma (2.8) follows that $\mathrm{x} \in \mathrm{T}(\mathrm{x}, \mathrm{y})$. Again by assumption (1)

$$
\mathrm{H}_{\mathrm{G}}\left(\mathrm{T}\left(y_{n}, x_{n}\right), \mathrm{T}(\mathrm{y}, \mathrm{x}), \mathrm{T}(\mathrm{y}, \mathrm{x})\right) \leq \mathrm{k} G\left(\left(y_{n}, x_{n}\right),(\mathrm{y}, \mathrm{x}),(\mathrm{y}, \mathrm{x})\right) \rightarrow 0 .
$$

Since $y_{n+1} \in \mathrm{T}\left(y_{n}, x_{n}\right)$ and $\lim _{\mathrm{n} \rightarrow \infty} G\left(y_{n+1}, \mathrm{y}, \mathrm{y}\right)=0$, it then by using lemma ( 2.8$)$ y $\in \mathrm{T}(\mathrm{y}, \mathrm{x})$. Hence $(\mathrm{x}, \mathrm{y})$ is a coupled fixed point of $\mathrm{T}$.

Corollary 3.2: Let $X$ be an ordered G-metric space and let T: $X$ $\times \mathrm{X} \rightarrow \mathrm{K}(\mathrm{X})$ satisfying:

1) There exists $\mathrm{k} \in(0,1)$ with

$$
\begin{aligned}
& H_{G}(T(x, y), T(u, v), T(u, v)) \leq \frac{k}{2} G((x, y),(u, v),(u, v)) \\
& \text { for all }(u, v) \preccurlyeq(x, y) \text {. }
\end{aligned}
$$

2) If $x_{1} \preccurlyeq x_{2}, y_{2} \preccurlyeq y_{1}, x_{i}, y_{i} \in \mathrm{X}(\mathrm{i}=1,2)$ then for all $u_{1} \in$ $\mathrm{T}\left(x_{1}, y_{1}\right)$ there exists $u_{2} \in \mathrm{T}\left(x_{2}, y_{2}\right)$ with $u_{1} \preccurlyeq u_{2}$, and for all $v_{1} \in \mathrm{T}\left(y_{1}, x_{1}\right)$ there exists $v_{2} \in \mathrm{T}\left(y_{2}, x_{2}\right)$ with $v_{2} \preccurlyeq v_{1}$ provided $G\left(\left(u_{1}, v_{1}\right),\left(u_{2}, v_{2}\right),\left(u_{2}, v_{2}\right)\right)<1$

3) There exists $x_{0}, y_{0} \in \mathrm{X}$, and some $x_{1} \in \mathrm{T}\left(x_{0}, y_{0}\right), y_{1} \in$ $\mathrm{T}\left(y_{0}, x_{0}\right)$ with $x_{0} \preccurlyeq x_{1}, y_{1} \preccurlyeq y_{0}$ such that $G\left(\left(x_{0}, y_{0}\right),\left(x_{1}\right.\right.$, $\left.\left.y_{1}\right),\left(x_{1}, y_{1}\right)\right)<1-\mathrm{k}$, where $\mathrm{k} \in(0,1)$.

4) If a non-decreasing sequence $x_{n} \rightarrow \mathrm{x}$ in $\mathrm{X}$, then $x_{n} \preccurlyeq \mathrm{x}$, for all $\mathrm{n}$ and if a non-increasing sequence $y_{n} \rightarrow \mathrm{y}$ in $\mathrm{X}$, then $\mathrm{y} \preccurlyeq$ $y_{n}$, for all $n$

Then $\mathrm{T}$ has a coupled fixed point.

As a consequence the above theorem also true for single valued mappings.

\section{Implicit condition}

For a nonempty subset $\mathrm{S}$ of the G-metric space $\mathrm{X}$, the diameter of $\mathrm{S}$ is defined as

$$
\delta_{G}(S)=\sup \{G(x, y, z): x, y, z \in S\} .
$$

If $\left\{y_{n}\right\}$ is a bounded sequence in G-metric space $\mathrm{X}$, let $j_{n}=\delta_{G}$ $\left(\left\{y_{n}, y_{n+1}, y_{n+2}, \ldots\right\}\right)$ for $\mathrm{n} \in \mathrm{N}$. Then $j_{n}<\infty$. for all $\mathrm{n} \in \mathrm{N}$, and $\left\{j_{n}\right\}$ is non-increasing and $j_{n} \geq 0$ for all $n \in \mathrm{N}$, and so there exists an $\mathrm{j} \geq 0$ such that $\lim _{\mathrm{n} \rightarrow \infty} j_{n}=\mathrm{j}$.

Theorem 4.1: Let $X$ be a complete bounded G-metric space and $T$ $: X \rightarrow X$ be a mapping such that for all $x, y, z \in X$,

$$
\phi(G(x, y, z), G(x, T x, z), G(y, T y, z), G(x, T y, z), G(y, T x, z),
$$

$$
G(T x, T y, T z)) \geq 0
$$

and $\phi: \mathrm{R}^{5}{ }_{+} \times \mathrm{R}+\rightarrow \mathrm{R}+$ be upper - semi continuous and be nondecreasing on $\mathrm{R}^{5}{ }_{+} \phi((\mathrm{u}, \mathrm{u}, \mathrm{u}, \mathrm{u}, \mathrm{u}), \mathrm{v}) \geq 0$ implies $\mathrm{v} \leq \psi(\mathrm{u})$, where $\psi: \mathrm{R}+\rightarrow \mathrm{R}+$ is a non-decreasing upper semi- continuous function with $\psi(0)=0$ and $\psi(\mathrm{t})<\mathrm{t}$ for $\mathrm{t}>0$, Then $\mathrm{T}$ has a unique fixed point $\mathrm{p}$ in $\mathrm{X}$ and $\mathrm{T}$ is continuous at $\mathrm{p}$.

Proof: Suppose that $x_{0}$ in $\mathrm{X}$ and $x_{n+1}=\mathrm{T} x_{n}$. Then the orbit $\left\{x_{n}\right\}$ is bounded.

Let

$$
j_{n}=\delta_{G}\left(\left\{x_{n}, x_{n+1}, x_{n+2}, \ldots\right\}\right), \mathrm{n} \in \mathrm{N} .
$$

from, the above remark $\lim _{\mathrm{n} \rightarrow \infty} j_{n}=\mathrm{j}$ for some $\mathrm{j} \geq 0$. If $x_{n+1}=x_{n}$ for some $\mathrm{n} \in \mathrm{N}$, then $\mathrm{T}$ has a fixed point, say $\mathrm{p} \in \mathrm{X}$.

Assume that $x_{n+1} \neq x_{n}$ for each $\mathrm{n} \in \mathrm{N}$. Let $\mathrm{k} \in \mathrm{N}$ be fixed Taking $\mathrm{x}=x_{n-1}, \mathrm{y}=x_{n+m-1}$ and $\mathrm{z}=x_{n+m+v-1}$ in (4.1) where $\mathrm{n} \geq \mathrm{k}$ and $\mathrm{m}, \mathrm{v} \in \mathrm{N}$, we have

$$
\begin{aligned}
& \phi\left(G\left(x_{n-1}, x_{n+m-1}, x_{n+m+v-1}\right), G\left(x_{n-1}, \mathrm{~T} x_{n-1}, x_{n+m+v-1}\right), G\left(x_{n+m-1},\right.\right. \\
& \left.\mathrm{T} x_{n+m-1}, x_{n+m+v-1}\right), G\left(x_{n-1}, \mathrm{~T} x_{n+m-1}, x_{n+m+v-1}\right), G\left(x_{n+m-1}, \mathrm{~T} x_{n-1},\right. \\
& \left.\left.\quad x_{n+m+v-1}\right), G\left(\mathrm{~T} x_{n-1}, \mathrm{~T} x_{n+m-1}, \mathrm{~T} x_{n+m+v-1}\right)\right) \\
& =\phi\left(G\left(x_{n-1}, x_{n+m-1}, x_{n+m+v-1}\right), G\left(x_{n-1}, x_{n}, x_{n+m+v-1}\right), G\left(x_{n+m-1},\right.\right. \\
& \left.\left.x_{n+m}, x_{n+m+v-1}\right), G\left(x_{n-1}, x_{n+m}, x_{n+m+v-1}\right), G\left(x_{n+m-1}, x_{n}, x_{n+m+v-1}\right)\right), \\
& \left.G\left(x_{n}, x_{n+m}, x_{n+m+v}\right)\right) \geq 0
\end{aligned}
$$

Thus we have

$$
\phi\left(\left(j_{n-1}, j_{n-1}, j_{n+m-1}, j_{n-1}, j_{n+m-1}\right), G\left(x_{n}, x_{n+m}, x_{n+m+v}\right)\right) \geq 0 .
$$

Since $\phi$ is non-decreasing on $\mathrm{R}^{5}$ and $\left\{j_{n}\right\}$ is non-increasing, we have

$$
\phi\left(\left(j_{k-1}, j_{k-1}, j_{k-1}, j_{k-1}, j_{k-1}\right), G\left(x_{n}, x_{n+m}, x_{n+m+v}\right)\right) \geq 0,
$$

which implies

$$
G\left(x_{n}, x_{n+m}, x_{n+m+v}\right) \leq \psi\left(j_{k-1}\right) .
$$

Taking limit sup over $\mathrm{n} \geq \mathrm{k}$, we have $j_{k} \leq \psi\left(j_{k-1}\right)$. Letting $\mathrm{k} \rightarrow \infty$, we get $\mathrm{j} \leq \psi(\mathrm{j})$.

If $\mathrm{j}>0$, then $\mathrm{j} \leq \psi(\mathrm{j})<\mathrm{j}$, which is a contradiction. Thus $\mathrm{j}=0$ and hence $\lim _{\mathrm{n} \rightarrow \infty} j_{n}=0$.

Thus given $\epsilon>0$, there exists $\mathrm{C} \in \mathrm{N}$ such that $j_{c}<\epsilon$.

Then we have for $\mathrm{n} \geq \mathrm{C}$ and $\mathrm{m}, \mathrm{v} \in \mathrm{N}, G\left(x_{n}, x_{n+m}, x_{n+m+v}\right)<\epsilon$. Therefore, $\left\{x_{n}\right\}$ is a G-Cauchy sequence in X. By the completeness of $\mathrm{X}$, there exists a $\mathrm{p} \in \mathrm{X}$ such that $\lim _{\mathrm{n} \rightarrow \infty} x_{n}=\mathrm{p}$.

Hence $\lim _{\mathrm{n} \rightarrow \infty} \mathrm{T} x_{n}=\mathrm{p}$.

Taking $\mathrm{x}=x_{n-1}, \mathrm{y}=x_{n+m-1}$ and $\mathrm{z}=\mathrm{p}$ in (4.1), we have

$$
\begin{gathered}
\phi\left(G\left(x_{n-1}, x_{n+m-1}, \mathrm{p}\right), G\left(x_{n-1}, \mathrm{~T} x_{n-1}, \mathrm{p}\right), G\left(x_{n+m-1}, \mathrm{~T} x_{n+m-1}, \mathrm{p}\right),\right. \\
\left.G\left(x_{n-1}, \mathrm{~T} x_{n+m-1}, \mathrm{p}\right), G\left(x_{n+m-1}, \mathrm{~T} x_{n-1}, \mathrm{p}\right), G\left(\mathrm{~T} x_{n-1}, \mathrm{~T} x_{n+m-1}, \mathrm{Tp}\right)\right) \\
=\phi\left(G\left(x_{n-1}, x_{n+m-1}, \mathrm{p}\right), G\left(x_{n-1}, x_{n}, \mathrm{p}\right), G\left(x_{n+m-1}, x_{n+m}, \mathrm{p}\right), G\left(x_{n-1},\right.\right. \\
\left.\left.\quad x_{n+m}, \mathrm{p}\right), G\left(x_{n+m-1}, x_{n}, \mathrm{p}\right), G\left(x_{n}, x_{n+m}, \mathrm{Tp}\right)\right) \geq 0 .
\end{gathered}
$$

Taking limit $\mathrm{n} \rightarrow \infty$, we have

$$
\phi G(p, p, p), G(p, p, p), G(p, p, p), G(p, p, p), G(p, p, p), G(p, p, T p)) \geq 0
$$

Which implies $G(p, p, T p) \leq \psi(G(p, p, p))=\psi(0)=0$. Hence $\mathrm{Tp}=\mathrm{p}$.

For the uniqueness, let $\mathrm{p}$ and $\mathrm{w}$ be fixed points of $\mathrm{T}$.

Taking $x=p, y=p$ and $z=w$ in (4.1), we have 


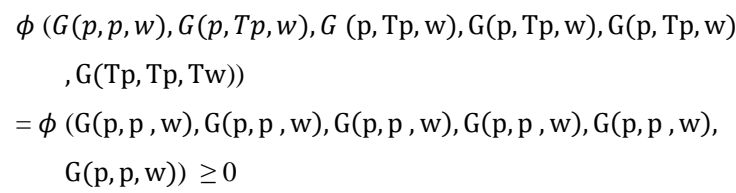

Which implies $G(p, p, w) \leq \psi(G(p, p, w))<G(p, p, w)$ which is a contradiction.

Thus, we have $\mathrm{p}=\mathrm{w}$.

Now, we show that $\mathrm{T}$ is continuous at $\mathrm{p}$.

Suppose that $\left\{y_{n}\right\}$ be a sequence in $X$ and $\lim _{n \rightarrow \infty} y_{n}=p$. Taking $x$ $=\mathrm{p}, \mathrm{y}=\mathrm{p}$ and $\mathrm{z}=\mathrm{y}_{\mathrm{n}}$ in (4.1), we have

$\phi\left(G\left(p, p, y_{n}\right), G\left(p, T p, y_{n}\right), G\left(p, T p, y_{n}\right), G\left(p, T p, y_{n}\right), G\left(p, T p, y_{n}\right)\right.$ G(Tp, Tp, Ty $\left.\left.{ }_{n}\right)\right)$

$=\phi\left(G\left(p, p, y_{n}\right), G\left(p, p, y_{n}\right), G\left(p, p, y_{n}\right), G\left(p, p, y_{n}\right), G\left(p, p, y_{n}\right)\right.$, $\left.\mathrm{G}\left(\mathrm{p}, \mathrm{p}, \mathrm{Ty}_{\mathrm{n}}\right)\right) \geq 0$

Which implies $G\left(p, p, T y_{n}\right) \leq \psi\left(G\left(p, p, y_{n}\right)\right)$.

Taking limit sup, we have

$\lim _{n \rightarrow \infty} G\left(p, p, \operatorname{Ty}_{n}\right) \leq \lim _{n \rightarrow \infty} \psi\left(G\left(p, p, y_{n}\right)\right) \leq \psi(0)=0$

Hence $\lim _{n \rightarrow \infty} T y_{n}=p=T p$ and hence $T$ is continuous at $p$.

\section{References}

[1] S.B. Nadler, Multi-valued Contraction Mappings, Pacific J Math 30(1969), pp. 475-478. http://dx.doi.org/10.2140/pjm.1969.30.475.

[2] L. Gorniewicz , Topological Fixed Point Theory of Multivalued Mappings, Kluwer Academic Publishers (1999)

[3] M.A. Al-Thagafi, N. Shahzad, Coincidence Point Generalized INonexpansive Multi-maps and Applications, Nonlinear Anal. 67(2007), pp. 2180-2188. http://dx.doi.org/10.1016/i.na.2006.08.042.

[4] Z. Mastafa and B. Sims, Fixed Point Theorems for Contractive Mappings in Complete G-Metric Spaces, Fixed point Theory and Applications, Vol. (2009), pp.10

[5] Z. Mustafa, (2005)," A New Structure for Generalized Metric Spaces with Applications to Fixed Point Theory", University of Newcastle, Newcastle, UK.

[6] Z. Mustafa, W. Shatanawi, M. Bataineh, Existence of Fixed Point Results in G-Metric Spaces , Int J Math Math Sci. (2009), pp. 10. http://dx.doi.org/10.1155/2009/283028.

[7] BE. Rhoades, M. Abbas, Common Fixed Point Results for NonCommuting Mappings without Continuity in Generalized Metric Spaces, Appl. Math. Comput. 215(2009), pp.262-269. http://dx.doi.org/10.1016/j.amc.2009.04.085.

[8] R. Saadati, SM. Vaezpour, P. Vetro, BE. Rhoades Fixed Point Theorems in Generalized Partially Ordered G-Metric Spaces, Math Comput. Model. 52(2010), pp. 797-801. http://dx.doi.org/10.1016/j.mcm.2010.05.009.

[9] A.C.M. Ran, M.C.B. Reurings, A Fixed Point Theorem in Partially Ordered Sets and Some Applacations to Matrix Equations, Proc. Amer. Math. Soc. 123(2004), pp.1435-1443. http://dx.doi.org/10.1090/S0002-9939-03-07220-4

[10] T.G. Bhaskar, V. Lakshmikantham, Fixed Point Theorems in Partially Ordered Metric Spaces and Applications, Nonlinear Anal. 65(2006), pp. 1379-1393. http://dx.doi.org/10.1016/j.na.2005.10.017

[11] V. Lakshmikantham, L. Ćirić, Coupled Fixed Point Theorems for Nonlinear Contractions in Partially Ordered Metric Spaces, Nonlinear Anal. 70(2009), pp.4341-4349. http://dx.doi.org/10.1016/j.na.2008.09.020

[12] S.K. Mohanta and S. Mohanta, A Common Fixed Point Theorem in G-Metric Spaces, CUBO A Mathematical Journal, Vol.14, (October 2012) No 03, pp.85-101

[13] N. Tahat, H. Aydi, E. Karapinar and W. Shatanawi, Common Fixed Points For Single-valued and Multi-valued Maps Satisfying A Generalized Contraction in G-Metric Spaces, Tahat et al. Fixed Point Theory and Applications,(2012) pp.48

[14] M. Abbasa, A. R. Khanb, T.Nazira, Common Fixed Point Of Multivalued Mappings In Ordered Generalized Metric Spaces, Filomat 26:5 ( 2012), pp.1045-1053. http://dx.doi.org/10.2298/FIL1205045A 